\title{
Criopreservación de Espermatozoides de Canino con Extracto de Hojas de Arándano (Vaccinium corymbosum L.)
}

\author{
Cryopreservation of Canine Spermatozoa with Blueberry Leaf Extract (Vaccinium corymbosum L.)
}

\author{
Mariana Deppe ${ }^{*, * *}$; Felipe Pezo*; Marjorie Reyes-Díaz ${ }^{* * * *}$ \& Jennie Risopatrón ${ }^{*, * * *}$
}

DEPPE, M; PEZO, F.; REYES-DÍAZ, M. \& RISOPATRÓN, J. Criopreservación de espermatozoides de canino con extracto de hojas de arándano (Vaccinium corymbosum L.). Int. J. Morphol., 34(2):653-659, 2016.

RESUMEN: La criopreservación espermática induce daño por estrés oxidativo en las células, lo que conlleva a un deterioro de la calidad del semen descongelado. Los espermatozoides pueden ser protegidos de este daño, por la adición de antioxidantes al medio de congelación. El objetivo de este estudio fue determinar el efecto de la adición de extracto de hojas de arándano (EHA) al medio de congelación, sobre la calidad de espermatozoides de canino criopreservados. Espermatozoides desprovistos del plasma seminal fueron congelados con diferentes concentraciones de EHA (0\%, control; $1 \%$, EHA1; $2 \%$, EHA2; $4 \%$, EHA4 y $6 \%$, EHA6) adicionadas al medio de congelación. Post descongelación se evalúo la motilidad progresiva; la viabilidad e integridad de la membrana plasmática (SYBR-14/PI) e integridad de la membrana acrosomal (FITC-PNA/PI) por citometría de flujo. La motilidad progresiva fue similar al control con las concentraciones de EHA1y EHA4 (P >0,05), mientras que con las concentraciones de EHA2 y EHA6 se observó una disminución significativa de este parámetro comparado con el control ( $\mathrm{P}<0,01$ y $\mathrm{P}<0,001$ respectivamente). La adición de EHA1, EHA2 y EHA4 al medio de congelación no presentó diferencias significativas respecto al control sobre la viabilidad e integridad de la membrana plasmática $(\mathrm{P}>0,05)$; por el contrario, con la adición de EHA6 se observaron valores significativamente menores $(\mathrm{P}<0,001)$. Los valores de integridad de la membrana acrosomal, con las diferentes concentraciones de EHA no presentaron diferencias significativas respecto al control. En conclusión, los resultados obtenidos en este estudio revelaron que las concentraciones de EHA utilizadas no fueron eficaces en mejorar la calidad del semen canino descongelado.

PALABRAS CLAVE: Canino; Espermatozoides; Criopreservación; Antioxidantes; Arándano.

\section{INTRODUCCIÓN}

Durante la criopreservación de semen, la congelación y posterior descongelación de éste conllevan a una baja calidad de los parámetros espermáticos en comparación al semen sin congelar, produciéndose cambios estructurales y funcionales de los espermatozoides. La criopreservación implica someter a los espermatozoides a situaciones que implican cambios drásticos de temperaturas (shock térmico), osmóticos y excesiva producción de especies reactivas de oxigeno (EROS) conducentes a estrés oxidativo. Entre los efectos perjudiciales de las EROS se describe la peroxidación lipídica de la membrana plasmática, alteraciones del acrosoma, cambios metabólicos, daños al ADN y disminución del potencial fecundante de los espermatozoides. En este contexto, numerosos estudios han utilizado sustancias con acción protectora (antioxidante), para prevenir o bloquear el daño oxidativo y mejorar la calidad del semen descongelado (Michael et al., 2007; Neagu et al., 2010; Daghigh-Kia et al., 2014).

En la especie canina el semen criopreservado presenta una baja sobrevida y una marcada reducción de la calidad con rangos de fecundación generalmente menores que con semen fresco. En esta especie los efectos perjudiciales de la criopreservación incluyen disminución de la motilidad, pérdida de la viabilidad e integridad de la membrana plasmática y acrosomal, afectándose además la translocación de fosfatidilserina, los niveles de peróxido de hidrógeno intracelular e integridad del ADN en los espermatozoides descongelados (Kim et al., 2010; Oettlé, 1986; Salinas et al., 2013). Se ha reportado que la adición de antioxidantes

\footnotetext{
Centro de Biotecnología en Reproducción (CEBIOR-BIOREN), Facultad de Medicina, Universidad de La Frontera, Temuco, Chile.

** Depto. de Ciencias Básicas, Facultad de Medicina, Universidad de La Frontera, Temuco, Chile.

*** Center of Plant, Soil Interaction and Natural Resources Biotechnology-BIOREN, Depto. de Ciencias Químicas y Recursos Naturales, Facultad de Ingeniería y Ciencias, Universidad de La Frontera, Temuco, Chile.

Financiamiento: Proyecto DI14-0011 de la Dirección de Investigación de La Universidad de La Frontera, Temuco, Chile, y Proyecto Fondecyt Regular 1120917.
} 
al medio de congelación ejercería un efecto positivo sobre la calidad del semen canino descongelado, mejorando la motilidad, viabilidad espermática e integridad de las membranas (Michael et al.; Monteiro et al., 2009; Neagu et al.; Gale et al., 2015).

Últimamente ha surgido un interés creciente en el uso de compuestos de origen vegetal, para ser adicionados a los medios de congelación en la criopreservación de espermatozoides. Se ha demostrado que la utilización de extractos obtenidos de algunas hierbas es beneficiosa para mejorar características espermáticas. Entre los extractos utilizados en la criopreservación de espermatozoides podemos mencionar: los extractos acuosos de raíz del ártico (Rhodiola sacra) que reducirían el proceso de peroxidación lipídica durante la congelación; extractos acuosos de romero (Rosmarinus officinalis) serían benéficos para la viabilidad y motilidad espermática, integridad acrosomal y funcionalidad de la membrana plasmática; el hinojo (Foeniculum vulgare) mejoraría la motilidad espermática y protegería contra la peroxidación lipídica. Sin embargo, el té verde (Camellia sinensis) no mejoraría la calidad de los espermatozoides descongelados (Zhao et al., 2009; Malo et al., 2010; Motlagh et al., 2014; Gale et al.).

Es altamente conocida la riqueza antioxidante que poseen los "berries", entre ellos Vaccinium corymbosum L. (arándano alto). Este arbusto, originario de Norteamérica, se ha adaptado muy bien a las condiciones edáficas y climáticas de la región centro sur de Chile, donde se ha establecido como un cultivo muy importante, debido a su alta riqueza en antioxidantes. Estudios de la actividad antioxidante (AA) en cultivares de esta especie han demostrado que esta actividad es mayor en hojas (InostrozaBlancheteau et al., 2014) que en frutos (Ribera et al., 2010), la cual está dada tanto por antioxidantes enzimáticos (principalmente SOD) como no enzimáticos (ácidos fenólicos, flavonoides y antocianinas) (Reyes Díaz et al., 2011; Inostroza-Blacheteau et al., 2014; Rojas-Lillo et al., 2014). En la actualidad no existen estudios que hayan evaluado el efecto de extracto de hojas de arándano (EHA) en semen de canino criopreservado o en otras especies. Por ello, el presente estudio determinó el efecto de la adición al medio de congelación espermática de diferentes concentraciones de EHA sobre la calidad de semen canino descongelado.

\section{MATERIAL Y MÉTODO}

El estudio fue aprobado por el Comité Ético Científico de la Universidad de La Frontera, Temuco, Chile. Todos los productos químicos utilizados fueron adquiridos de
Sigma (St. Louis MO, USA), a menos que se indique lo contrario. Todas las soluciones se prepararon utilizando agua de un sistema Milli-Q Síntesis (Millipore, Bedford, MA, USA).

Lugar del estudio. Los experimentos conducentes a obtener los extractos de hojas de arándano, fueron realizados en el Centro de Interacción Suelo Planta y Biotecnología de Recursos Naturales (BIOREN-UFRO) de la Universidad de La Frontera. Los ensayos experimentales de criopreservación de espermatozoides se realizaron en el Centro de Biotecnología en Reproducción (CEBIOR-BIOREN), perteneciente a la Facultad de Medicina de la Universidad de La Frontera.

\section{Obtención de extracto acuoso de hojas de arándano}

(EHA). Se utilizaron hojas de arándano alto del cultivar comercial Legacy. Para la obtención del EHA acuoso se realizó una infusión según lo reportado por Malo et al. Brevemente, $0,15 \mathrm{~g}$ de hojas fueron añadidas a $100 \mathrm{~mL}$ de agua calentada a $100{ }^{\circ} \mathrm{C}$ y mantenidas a esta temperatura por 10 min. Una vez que el agua alcanzó los $25^{\circ} \mathrm{C}$, la solución fue filtrada para remover las hojas.

Animales. Como donantes de semen fueron utilizados cinco caninos adultos (Canis lupus familiaris), raza Chihuahua, con peso promedio de $2.5 \pm 0.5 \mathrm{~kg}$, y cuyas edades fluctuaron entre 1 a 4 años. Los animales pertenecientes a criadores privados fueron entrenados previamente como donantes de semen y durante la etapa experimental se mantuvieron por un periodo mínimo de abstinencia de tres días entre cada colecta de semen. La condición sanitaria de los donantes fue evaluada periódicamente por un médico veterinario y fueron mantenidos con una dieta balanceada y agua $a d$ libitum.

Obtención y evaluación de los eyaculados. En cada ensayo experimental $(n=15)$, las segundas fracciones ricas en espermatozoides de los eyaculados fueron obtenidas por manipulación digital en tubos graduados previamente temperados. Las muestras fueron analizadas con respecto a motilidad progresiva (ver en evaluaciones de parámetros espermáticos) y concentración espermática. La concentración espermática fue determinada utilizando un fotómetro SDM1 (SpermaCue, Minitüb, Tiefenbach, Germany). Sólo muestras con una concentración $\geq 200 \times 10^{6}$ espermatozoides/ $\mathrm{mL}$ y una motilidad $\geq 70 \%$ fueron incluidas en el estudio (Kim et al.). Posteriormente, alícuotas similares en concentración espermática fueron mezcladas para obtener un pool de semen con el propósito de aumentar el volumen y eliminar la variabilidad entre las muestras a evaluar.

Criopreservación de espermatozoides de canino con medios de congelación suplementados con diferentes con- 
centraciones de EHA: El procedimiento de congelación y descongelación espermática se realizó de acuerdo al protocolo descrito previamente por Kim et al., con modificaciones. En cada ensayo experimental el pool de semen fue dividido en cinco alícuotas iguales, las cuales fueron centrifugadas a $300 \mathrm{~g}$ por $5 \mathrm{~min}$, el plasma seminal eliminado y el pellet de cada alícuota resuspendido en Tris (Tris buffer-glucosa) y nuevamente centrifugadas. El sobrenadante fue eliminado y cada pellet resuspendido (200x $10^{6}$ espermatozoides $/ \mathrm{mL}$ ) en el medio de congelación 1 (Tris suplementado con $20 \%$ [v/v] de yema de huevo y 3 $\%$ [v/v] de glicerol) suplementado con diferentes concentraciones de EHA y sin suplementación (control). Las concentraciones de EHA adicionadas al medio de congelación 1 fueron de un $1 \%(\mathrm{v} / \mathrm{v})$ de EHA (EHA1), $2 \%(\mathrm{v} / \mathrm{v})$ de EHA (EHA2), $4 \%$ (v/v) de EHA (EHA4), $6 \%$ (v/v) de EHA (EHA6) y sin EHA como control. Posteriormente las muestras espermáticas fueron equilibradas a $4^{\circ} \mathrm{C}$ por $1 \mathrm{~h}$ y luego cada muestra fue rediluida $(1: 1 \mathrm{v} / \mathrm{v})$ con el medio de congelación 2 [Tris suplementado con $20 \%$ [v/v] yema de huevo, $7 \%$ [v/v] glicerol y $1 \%$ [v/v] de Equex STM paste (Nova Chemical Sales, Scituate, MA, USA)]. La concentración final de espermatozoides correspondió a $100 \times 10^{6}$ espermatozoides $/ \mathrm{mL}$. Transcurridos $10 \mathrm{~min}$ a $4{ }^{\circ} \mathrm{C}$ de equilibrio con el medio de congelación 2, las muestras espermáticas fueron guardadas en pajuelas de $0,5 \mathrm{~mL}$ y colocadas en atmósfera de vapor de nitrógeno líquido $\left(\mathrm{LN}_{2}\right)$ de manera horizontal, $4 \mathrm{~cm}$ sobre la superficie del $\mathrm{LN}_{2} \mathrm{du}-$ rante 10 minutos y posteriormente sumergidas y almacenadas en $\mathrm{LN}_{2}$ a lo menos por un mes. Cada pajuela fue descongelada en un baño de agua a $38^{\circ} \mathrm{C}$ por $60 \mathrm{~s}$ y el contenido de cada una vaciado cuidadosamente en diferentes tubos con medio de descongelación [Tris Buffer a un volumen de 1:2 (v/v)] y centrifugando a $300 \mathrm{~g}$ por $5 \mathrm{~min}$, el sobrenadante fue eliminado y el pellet resuspendido en medio Tris Buffer a una concentración de $2 \times 10^{6}$ espermatozoides $/ \mathrm{mL}$. Alícuotas de suspensión espermática de cada uno de los tubos fueron utilizadas para la evaluación de los parámetros espermáticos correspondientes.

Evaluación de parámetros espermáticos post-descongelación: Los espermatozoides congelados con y sin EHA fueron analizados post-descongelación con respecto a motilidad progresiva, viabilidad e integridad de la membrana plasmática (VIMP) e integridad de la membrana acrosomal (IMA).

Motilidad Progresiva: La motilidad progresiva fue determinada subjetivamente utilizando un microscopio de luz (Carl Zeiss, Jena, Germany) con aumento de 400X. Diez microlitros de cada suspensión espermática fueron colocados sobre un portaobjeto temperado y el porcentaje de espermatozoides con motilidad progresiva fue determinado por observación en un mínimo de 300 células, en seis cam- pos diferentes. El promedio de seis estimaciones sucesivas fue registrado como porcentaje de motilidad progresiva (Risopatrón et al., 2002; Kim et al.).

Viabilidad e Integridad de Membrana plasmática: La evaluación de los espermatozoides vivos con integridad de la membrana plasmática se realizó con la técnica de tinción fluorescente SYBR-14/PI (LIVE/DEAD ${ }^{\circledR}$ Sperm Viability Kit, Molecular Probes L-7011, Eugene, OR, USA), de acuerdo a lo descrito por Cheuqueman et al. (2012) con modificaciones. A un volumen de $250 \mu 1$ de suspensión espermática ( $2 \times 10^{6}$ espermatozoides $/ \mathrm{mL}$ ) se adicionaron $1 \mu \mathrm{l}$ de SYBR14 (10 mM en DMSO), luego de 7 min de incubación a 38 ${ }^{\circ} \mathrm{C}$, se adicionó $2 \mu \mathrm{l}$ de Ioduro de Propidio (PI, $12 \mathrm{mM}$ ) incubando 7 min adicionales a $38{ }^{\circ} \mathrm{C}$ y lavando las suspensiones espermáticas por centrifugación a $300 \mathrm{x}$ g por $5 \mathrm{mi}-$ nutos con $1 \mathrm{~mL}$ de Tris. Luego de eliminar el sobrenadante el pellet fue resuspendido en $400 \mu \mathrm{l}$ del mismo medio para su análisis por citometría de flujo. Los espermatozoides analizados se clasificaron como vivos con membrana plasmática intacta (SYBR-14 +/PI -), muertos (SYBR-14 - /PI +) y moribundos (SYBR-14 + /PI +).

Integridad de la membrana acrosomal: La integridad de la membrana del acrosoma fue evaluada en espermatozoides vivos, utilizando la técnica de tinción fluorescente de fluoresceína conjugada con Lectina de Arachis hypogaea (peanut, FITC-PNA, L-7381, Sigma-Aldrich Co., St. Louis MO, USA) y PI. El procedimiento se realizó en base a lo descrito previamente por Kim et al. (2012) con modificaciones. A alícuotas de $250 \mu \mathrm{l}$ de suspensión espermática (2x10 $10^{6}$ espermatozoides $/ \mathrm{mL}$ ) se adicionó $1 \mu 1 \mathrm{PI}(250 \mathrm{mg} /$ $\mathrm{mL})$ y $0,5 \mu \mathrm{l}$ FITC-PNA $(100 \mu \mathrm{g} / \mathrm{mL})$. Luego de incubar a $38^{\circ} \mathrm{C}$ por 15 min se adicionó $1 \mathrm{~mL}$ de Tris centrifugando a $300 \mathrm{~g}$ por $5 \mathrm{~min}$ y eliminando el sobrenadante. La muestra se resuspendió en $400 \mu \mathrm{l}$ de Tris para su análisis por citometría de flujo. Los espermatozoides fueron clasificados como vivos con acrosoma intacto (FITC-PNA- /PI-) y vivos con acrosoma dañado (FITC-PNA+/PI-).

Citometría de flujo y obtención de datos: Las lecturas de fluorescencia de los espermatozoides tratados con cada una de las tinciones específicas se realizaron en un citómetro de flujo FACS Canto II, Becton Dickinson, USA. Las células fueron excitadas a una longitud de onda de $488 \mathrm{~nm}$ utilizando un laser de Argón. Cada análisis incluyó 10.000 espermatozoides teñidos, los cuales fueron cuantificados simultáneamente para cada fluorescencia. Los datos fueron proporcionados sobre una escala logarítmica y digitalizados utilizando el software FACs DiVa 6.0, Becton Dickinson, USA.

Análisis estadísticos. Para el análisis de datos se utilizó el software GraphPad Prism ${ }^{\circledR}$ versión 5.0 (GraphPad Software, 
San Diego, CA, USA). Los resultados fueron sometidos a un test de normalidad para detectar distribución Gaussiana. La prueba de análisis de la varianza (ANOVA) se aplicó para cada evaluación, con el fin de comprobar la existencia de diferencias significativas en los diferentes grupos experimentales. El test paramétrico de comparación múltiple de Tuckey fue utilizado para establecer diferencias significativas entre los diferentes grupos para cada una de los parámetros espermáticos evaluados. Los resultados son presentados como promedio $\pm \mathrm{DE}$, con niveles de significancia correspondientes a $\mathrm{P}<0,05$.

\section{RESULTADOS}

Efecto de diferentes concentraciones de EHA sobre la motilidad progresiva: El efecto de la adición de diferentes concentraciones de EHA al medio de congelación sobre la motilidad progresiva en los espermatozoides de canino es presentado en la Figura 1a. No fue observado un efecto positivo sobre la motilidad espermática con la adición de EHA en ninguna de las concentraciones utilizadas con respecto al control. Los grupos EHA1 y EHA4 no presentaron diferencia significativa respecto al control $(54,6 \%$ y $53,8 \%$ vs. $56,8 \% ; \mathrm{P}>0,05)$, mientras que en los grupos EHA2 y EHA6 fueron observados valores de motilidad significativamente menores que en el control $(50,8 \%$ y $47,6 \%$; $\mathrm{P}<0,01$ y $\mathrm{P}$ $<0,001$, respectivamente).

Efecto de diferentes concentraciones de EHA sobre la viabilidad e integridad de la membrana plasmática: Los resultados de la adición de diferentes concentraciones de EHA sobre la viabilidad e integridad de la membrana plasmática de los espermatozoides de canino post-descongelación son presentados en la Figura 1b. Ninguna de las concentraciones de EHA fue eficaz en mejorar significativamente los valores de VIMP en los espermatozoides descongelados. Los grupos experimentales EHA1, EHA2, EHA4 y el control no presentaron diferencia significativa entre sí, con valores de VIMP de 55,3 $\%, 51,2 \%, 52,0 \%$ y 54,7\%, respectivamente (P >0,05); mientras que el grupo EHA6 presentó los valores más bajos en este parámetro espermático $(47,3 \%)$ y significativamente menores a los del control y grupo EHA1 $(\mathrm{P}<0,001)$.

Efecto de diferentes concentraciones de EHA sobre la integridad de la membrana acrosomal: La calidad espermática en términos de integridad de la membrana acrosomal son presentados en la Figura 1c. En este parámetro espermático no fueron observadas diferencias estadísticamente significativas en ninguno de los grupos experimentales con EHA, respecto al control sin EHA. Los

espermatozoides congelados con EHA6 presentaron los menores valores de IMA y al contrario los espermatozoides congelados con EHA4 presentaron los mayores valores de IMA, siendo éstos significativamente diferentes entre sí (48,5 $\%$ y $55,7 \%$, P <0,01). Los valores de IMA observados en los grupos EHA1, EHA2 y el control fueron de 53,8 \%, 51,5 $\%$ y $53,8 \%$, respectivamente.
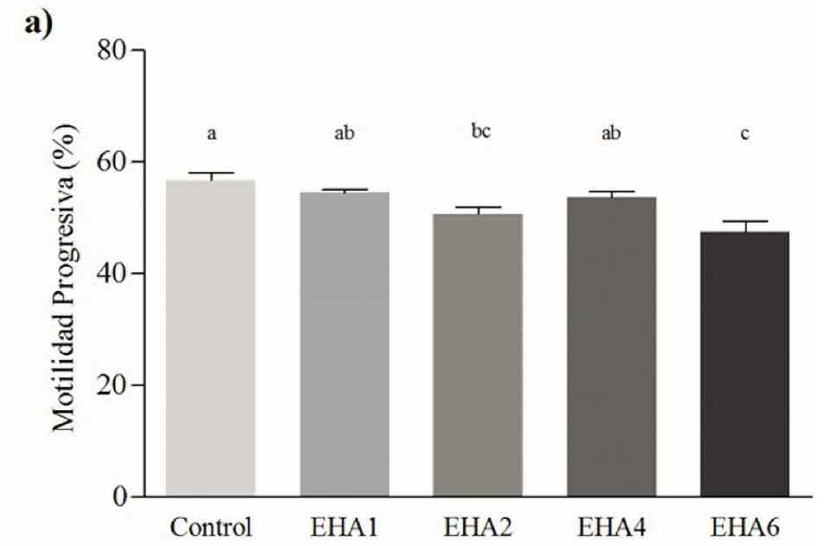

b)
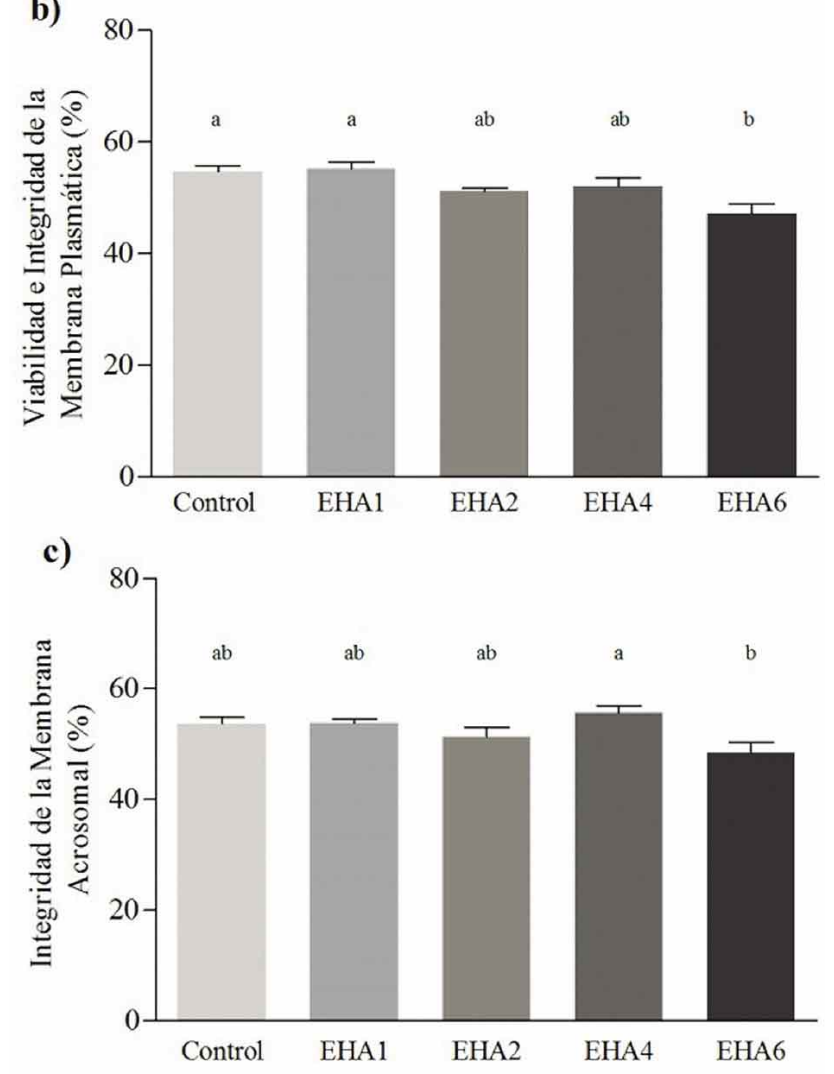

Fig. 1. Espermatozoides de canino congelados con diferentes concentraciones de extracto de hojas de arándano (EHA) adicionadas al medio de congelación (Promedio \pm DE). a) Motilidad progresiva, b) Viabilidad e integridad de la membrana plasmática y c) Integridad de la membrana acrosomal. EHA (0 \%; Control), EHA1(1 $\%)$, EHA2 (2\%), EHA4 (4\%) y EHA6 (6\%). Diferentes letras indican diferencias estadísticamente significativas $(\mathrm{P}<0,05)$. 


\section{DISCUSIÓN}

El semen criopreservado presenta una baja sobrevida y una marcada reducción de su calidad con rangos de fecundación generalmente menores que con semen fresco. Se ha reportado que la adición de antioxidantes al medio de congelación ejerce un efecto positivo sobre la calidad del semen canino descongelado, mejorando la motilidad, viabilidad espermática e integridad de las membranas (Michael et al.; Monteiro et al.; Neagu et al.).

Hasta la fecha, no existe información disponible del efecto del extracto de hojas de arándano en la criopreservación de espermatozoides de mamífero, y este estudio sería el primero que evalúa la suplementación del medio de congelación con EHA en espermatozoides de canino, por lo cual no existen antecedentes que nos permitan comparar nuestros resultados. En nuestro estudio, la adición de extractos de hojas de arándano al medio de congelación, con las concentraciones de EHA utilizadas no mejoró la motilidad espermática del semen de canino descongelado. Escasos estudios existen en espermatozoides de canino criopreservados con antioxidantes de origen vegetal. González et al. (2010), estudiaron la motilidad espermática en canino adicionando romero al medio de congelación la que se redujo significativamente, especialmente con la concentración más alta del extracto de romero. Este resultado concuerda con nuestro estudio en que la concentración mayor de EHA fue la más perjudicial. Estudios previos, en espermatozoides de otras especies donde se ha adicionado componentes de origen vegetal al medio de congelación han demostrado diferentes efectos sobre la motilidad inmediatamente post-descongelación. $\mathrm{Al}$ respecto, el uso de romero en espermatozoides de cerdo (Malo et al., 2010), carnero (Mascaro et al., 2013) y toro (Daghigh-Kia et al.), no tendría efecto sobre la motilidad progresiva. Contrariamente, Motlagh et al., observó en carnero que la utilización de romero en el medio de congelación mejoraría significativamente la motilidad progresiva inmediatamente después de descongelar. De igual modo, la motilidad progresiva en espermatozoides de carnero mejoró con adición de clavo de olor (Syzygium aromaticum) y en cerdo el hinojo aumentó la motilidad total (Baghshahi et al., 2014; Malo et al., 2012).

En este estudio ninguna de las concentraciones de EHA utilizadas fue efectiva en mejorar la viabilidad e integridad de la membrana plasmática, y la mayor concentración disminuyó significativamente este parámetro. En canino, González et al., describe que el uso de concentraciones crecientes de romero serían inadecuadas para la criopreservación, afectándose negativamente la viabilidad e integridad de la membrana plasmática. Similarmente, nues- tros resultados mostraron los menores rangos de este parámetro con la mayor concentración de EHA. De igual modo el romero e hinojo no presentan efectos significativos respecto a la viabilidad espermática en espermatozoides de cerdo inmediatamente descongelados (Malo et al., 2011, 2012). Por otro lado, el uso de té verde en esta especie no mejoró la viabilidad e integridad de la membrana (Gale et al.). Contrario a nuestros resultados, en espermatozoides de toro y carnero, en que se evaluó el efecto del romero y clavo de olor respectivamente, sobre la viabilidad espermática e integridad de la membrana, se demostró que éstos fueron efectivos en mejorar este parámetro post descongelación (Daghigh-Kia et al.; Baghshahi et al.).

Respecto a la integridad de la membrana acrosomal, en este estudio se observó que los EHA no tendrían efecto sobre este parámetro, ya que ninguna de las concentraciones de EHA utilizadas produjo aumento o disminución significativa en los porcentajes de integridad de la membrana acrosomal en los espermatozoides descongelados. En canino, González et al., observaron que el uso de romero no sería beneficioso para mantener la integridad acrosomal, pues los valores obtenidos post-descongelación fueron significativamente menores que sin la adición de romero. Similares resultados a nuestro estudio obtuvieron Motlagh et al. y Malo et al. (2011) al adicionar extractos acuosos de romero al medio de congelación de carnero y cerdo, en donde no hubo efecto sobre el estado acrosomal de los espermatozoides descongelados. Igualmente, el uso de hinojo y té verde en cerdo no mejoraría la integridad acrosomal (Malo et al.; Gale et al.).

Del presente estudio puede concluirse que la adición de extracto de hojas de arándano en las concentraciones utilizadas no es un suplemento adecuado para adicionar al medio de congelación en espermatozoides de canino, en relación a las funciones espermáticas evaluadas.

DEPPE, M; PEZO, F.; REYES-DÍAZ, M. \& RISOPATRÓN, J. Cryopreservation of canine spermatozoa with blueberry leaf extract (Vaccinium corymbosum L.). Int. J. Morphol., 34(2):653$659,2016$.

SUMMARY: During cryopreservation, oxidative stress damage leads to a deterioration of the quality of thawed semen, which could be reduced by the addition of antioxidants to freezing extender. This study was designed to determine the effect of the addition of blueberry leaf extract (EHA) to freezing extender, on quality of cryopreserved canine sperms. Sperm devoid from seminal plasma were frozen with different concentrations of EHA $(0 \%$, control; $1 \%$, EHA $1 ; 2 \%$, EHA2; $4 \%$, EHA4 y $6 \%$, EHA6) added to freezing extender. Post-thawing progressive motility was evaluated; the viability and plasma membrane integrity (SYBR- 
14/PI) and acrosomal membrane integrity (FITC-PNA/PI) were assessed by flow cytometry. Progressive motility was similar to the control with concentrations of EHA1 and EHA4 ( $\mathrm{P}>0.05)$; at concentrations of EHA2 and EHA6 a significant decrease of this parameter compared to control ( $\mathrm{P}<0.01$ and $\mathrm{P}<0.001$, respectively) was observed. The addition of EHA1, EHA2 and EHA4 to the freezing extender showed no significant differences with respect to the control on viability and plasma membrane integrity $(\mathrm{P}>0.05)$; however with the addition of EHA6 values significantly lower $(\mathrm{P}$ $<0.001)$ were exhibited. The concentrations of EHA used showed no significant differences with respect to the control on acrosome membrane integrity. In conclusion, the results of this study revealed that none of the concentrations of EHA used were effective in improving canine thawed semen quality.

KEY WORDS: Canine; Spermatozoa; Cryopreservation; Antioxidants; Blueberry.

\section{REFERENCIAS}

Baghshahi, H.; Riasi, A.; Mahdavi, A. H. \& Shirazi, A. Antioxidant effects of clove bud (Syzygium aromaticum) extract used with different extenders on ram spermatozoa during cryopreservation. Cryobiology, 69(3):482-7, 2014.

Cheuquemán, C.; Bravo, P.; Treulén, F.; Giojalas, L.; Villegas, J.; Sánchez, R. \& Risopatrón, J. Sperm membrane functionality in the dog assessed by flow cytometry. Reprod. Domest. Anim., 47(1):39-43, 2012.

Daghigh-Kia, H.; Olfati-Karaji, R.; Hoseinkhani, A. \& Ashrafi, I. Effect of rosemary (Rosmarinus officinalis) extracts and glutathione antioxidants on bull semen quality after cryopreservation. Span. J. Agric. Res., 12(1):98-105, 2014.

Gale, I.; Gil, L.; Malo, C.; González, N. \& Martínez, F. Effect of Camellia sinensis supplementation and increasing holding time on quality of cryopreserved boar semen. Andrologia, 47(5):505-12, 2015.

González, N.; Gil, L.; Martínez, F.; Malo, C.; Cano, R.; Mur, P. $\&$ Espinosa, E. Effect of natural antioxidant rosemary in canine soya freezing extender. Reprod. Domest. Anim., 45:88, 2010.

Inostroza-Blancheteau, C.; Reyes-Díaz, M.; Arellano, A.; Latsague, M.; Acevedo, P.; Loyola, R.; Arce-Johnson, P. \& Alberdi, M. Effects of UV-B radiation on anatomical characteristics, phenolic compounds and gene expression of the phenylpropanoid pathway in highbush blueberry leaves. Plant Physiol. Biochem, 85:85-95, 2014.

Kim, S. H.; Yu, D. H. \& Kim, Y. J. Effects of cryopreservation on phosphatidylserine translocation, intracellular, hydrogen peroxide, and DNA integrity in canine sperm. Theriogenology, 73:282-92, 2010.
Kim, S.; Lee, Y.; Yang, H. \& Kim, Y. J. Rapid freezing without cooling equilibration in canine sperm. Anim. Reprod. Sci., 130(1-2):111-8, 2012.

Malo, C.; Gil, L.; Gonzalez, N.; Martínez, F.; Cano, R.; de Blas, I. \& Espinosa, E. Anti-oxidant supplementation improves boar sperm characteristics and fertility after cryopreservation: comparison between cysteine and rosemary (Rosmarinus officinalis). Cryobiology, 61(1):142-7, 2010.

Malo, C.; Gil, L.; Cano, R.; Martínez, F. \& Galé, I. Antioxidant effect of rosemary (Rosmarinus officinalis) on boar epididymal spermatozoa during cryopreservation. Theriogenology, 75(9):1735-41, 2011.

Malo, C.; Gil, L.; Cano, R.; González, N. \& Luño, V. Fennel (Foeniculum vulgare) provides antioxidant protection for boar semen cryopreservation. Andrologia, 44(Suppl. 1):710$5,2012$.

Mascaro, F.; Gil, L.; Malo, C.; Gonzales, N.; Martínez, F. \& de Blas, I. Effect of pasteurized egg and Rosmarinus officinalis supplementation on quality of cryopreserved ram semen. Cryo Letters, 34(4):422-31, 2013.

Michael, A.; Alexopoulos, C.; Pontiki, E.; Hadjipavlou-Litina, D.; Saratsis, P. \& Boscos, C. Effect of antioxidant supplementation on semen quality and reactive oxygen species of frozen-thawed canine spermatozoa. Theriogenology, 68(2):204-12, 2007.

Monteiro, J. C.; Gonçalves, J. S., Rodrigues, J. A.; Lúcio, C. F.; Silva, L. C.; Assumpção, M. E. \& Vannucchi, C. I. Influence of ascorbic acid and glutathione antioxidants on frozenthawed canine semen. Reprod. Domest. Anim., 44(Suppl. 2):359-62, 2009.

Motlagh, M. K.; Sharafi, M.; Zhandi, M.; MohammadiSangcheshmeh, A.; Shakeri, M.; Soleimani, M. \& Zeinoaldini, S. Antioxidant effect of rosemary (Rosmarinus officinalis L.) extract in soybean lecithin-based semen extender following freeze-thawing process of ram sperm. Cryobiology, 69(2):217-22, 2014.

Neagu, V. R.; García, B. M.; Sandoval, C. S.; Rodríguez, A. M.; Ferrusola, C. O.; Fernández, L. G.; Tapia, J. A. \& Peña, F. J. Freezing dog semen in presence of the antioxidant butylated hydroxytoluene improves postthaw sperm membrane integrity. Theriogenology, 73(5):645-50, 2010.

Oettlé, E. E. Changes in acrosome morphology during cooling and freezing of dog semen. Anim. Reprod. Sci., 12(2):14550, 1986.

Reyes-Díaz, M.; Meriño-Gergichevich, C.; Alarcón, E.; Alberdi, M. \& Horst, W. J. Calcium sulfate ameliorates the effect of aluminum toxicity differentially in genotypes of highbush blueberry (Vaccinium corymbosum L.). J. Soil Sci. Plant Nutr., 11(4):59-78, 2011. 
DEPPE, M; PEZO, F.; REYES-DÍAZ, M. \& RISOPATRÓN, J. Criopreservación de espermatozoides de canino con extracto de hojas de arándano (Vaccinium corymbosum L.). Int. J. Morphol., 34(2):653-659, 2016.

Ribera, A.; Reyes-Díaz, M.; Alberdi, M.; Zuñiga, G. E. \& Mora, M. L. Antioxidant compounds in skin and pulp of fruits change among genotypes and maturity stages in highbush blueberry (Vaccinium corymbosum L.) grown in Southern Chile. J. Soil Sci. Plant Nutr., 10(4):509-36, 2010.

Risopatrón, J.; Catalán, S.; Miska, W.; Schill, W. B. \& Sánchez, R. Effect of albumin and polyvinyl alcohol on the vitality, motility and acrosomal integrity of canine spermatozoa incubated in vitro. Reprod. Domest. Anim., 37(6):347-51, 2002.

Rojas-Lillo, Y.; Alberdi, M.; Acevedo, P.; Inostroza-Blancheteau, C.; Rengel, Z.; Mora, M. L. \& Reyes-Díaz, M. Manganese toxicity and UV-B radiation differentially influence the physiology and biochemistry of highbush blueberry (Vaccinium corymbosum) cultivars. Func. Plant Biol., 41(2):156-167, 2014.

Salinas, P.; Sánchez, R. \& Risopatrón, J. Cryopreservation of Canine Spermatozoa at $-80^{\circ}$ C. Int. J. Morphol., 31(1):217-24, 2013.

Zhao, H. W.; Li, Q. W.; Ning, G. Z.; Han, Z. S.; Jiang, Z. L. \& Duan, Y. F. Rhodiola sacra aqueous extract (RSAE) improves biochemical and sperm characteristics in cryopreserved boar semen. Theriogenology, 71(5):849-57, 2009.

\author{
Dirección para correspondencia: \\ Mariana Deppe A. \\ Depto. Ciencias Básicas \\ Facultad de Medicina \\ Universidad de La Frontera \\ Casilla 54-D \\ Temuco \\ CHILE
}

Email: mariana.deppe@ufrontera.cl

Recibido: 11-03-2016

Aceptado: 29-03-2016 